\title{
Comparison of accuracy in three versions of simplified sequential organ failure assessment scores to predict prognosis of septic patients
}

\author{
qifang shi ${ }^{1}$, Ying $\mathrm{Xu}^{1}$, Bing-yu Zhang ${ }^{2}$, Wei $\mathrm{Qu}^{3}$, Shu-yun Wang ${ }^{1}$, Wen-long Zheng ${ }^{1}$, and \\ Ying sheng ${ }^{1}$ \\ ${ }^{1}$ Shanghai Pudong Hospital \\ ${ }^{2}$ Gongli Hospital Affiliated to Naval Medical University \\ ${ }^{3}$ Shanghai University of Medicine \& Health Sciences Affiliated Zhoupu Hospital
}

February 1, 2021

\begin{abstract}
Background: Evidence shows that simplified SOFA scoring system has better clinical practice. Objective: This study aimed to compare the scores acquired with the simplified sequential organ failure assessment (sSOFA), simplified organ dysfunction criteria optimized for electronic health records (eSOFA), and simplified and accurate sequential organ failure assessment (saSOFA) for their accuracies in predicting the prognosis of septic patients. Methods: This retrospective observational study was conducted at three major academic hospitals. Clinical data from 574 patients diagnosed with sepsis following the Third International Consensus Definitions for Sepsis and Septic Shock (Sepsis-3)were retrospectively retrieved and analyzed. Scores from the quick sequential organ failure assessment (qSOFA) and sequential organ failure assessment (SOFA) were used as reference scores. The area under the receiver operating characteristic curve (AUROC) was used to compare the accuracies of eSOFA, sSOFA, and sa-SOFA scores in predicting in-hospital mortality. Results: AUROC analysis demonstrated the predictability of the five scoring systems for sepsis surveillance, listed in descending order as: sa-SOFA,0.790 (95\% confidence interval [CI]: 0.754-0.822); SOFA, 0.774 (95\% CI: 0.738-0.808); eSOFA,0.729 (95\% CI: 0.691-0.765); sSOFA,0.681 (95\% CI: 0.641- 0.719); and qSOFA,0.618(95\% CI: 0.577-0.658). Moreover, sa-SOFA and SOFA scores $(\mathrm{Z}=1.950, \mathrm{p}=0.051)$ did not significantly differ from each other in discriminatory power, but the sa-SOFA score had a higher power than either the sSOFA or eSOFA scores ( $\mathrm{p}$ values $<0.001$ ). Conclusion: sa-SOFA showed the highest accuracy in predicting in-hospital fatality of septic patients when compared with sSOFA and eSOFA.
\end{abstract}

\section{Hosted file}

Main Manuscrip.pdf available at https://authorea.com/users/392991/articles/506732-comparisonof-accuracy-in-three-versions-of-simplified-sequential-organ-failure-assessment-scoresto-predict-prognosis-of-septic-patients 

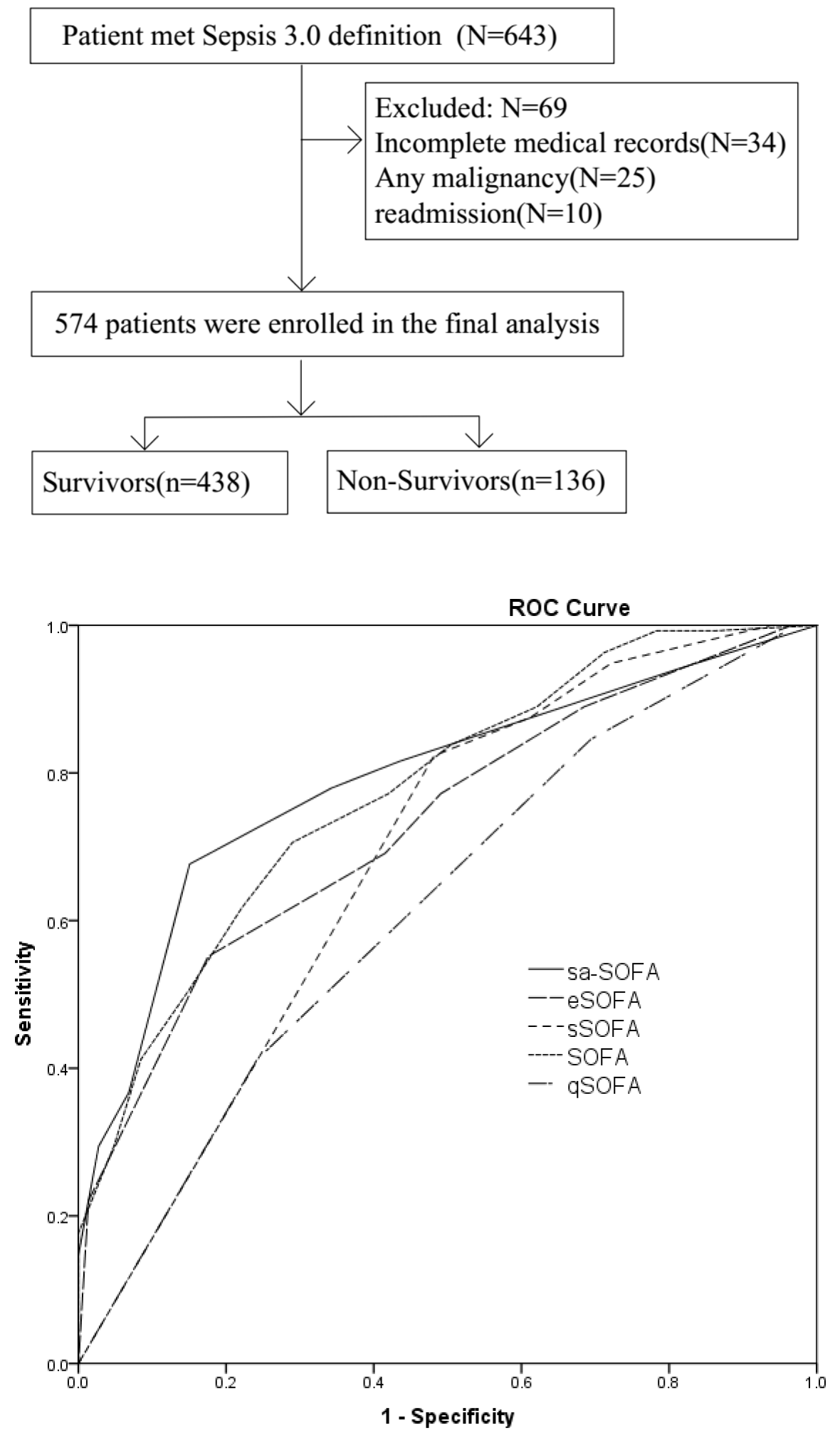

Diagonal segments are produced by ties. 